\title{
Infiltrative invasion of the diaphragm: an uncommon manifestation of recurrent hepatocellular carcinoma A retrospective cohort study
}

\author{
Chu Hyun Kim ${ }^{1}$, Kyoung Doo Song ${ }^{1}$, Jung Han Woo ${ }^{2}$ \\ ${ }^{1}$ Department of Radiology and Center for Imaging Science, Samsung Medical Center, Sungkyunkwan University School of \\ Medicine, Seoul, Korea \\ ${ }^{2}$ Department of Radiology, Seoul District Hospital, Seoul, Korea
}

Received: July 6, 2021

Revised: July 22, 2021

Accepted: July 27, 2021

Corresponding author:

Kyoung Doo Song

Department of Radiology and

Center for Imaging Science,

Samsung Medical Center,

Sungkyunkwan University

School of Medicine, 81 Irwon-

ro, Gangnam-gu, Seoul 06351, Korea

Tel: +82-2-3410-2519

E-mail: kd3893.song@samsung. com

\begin{abstract}
Purpose: To report on infiltrative invasion of the diaphragm, an uncommon manifestation of recurrent hepatocellular carcinoma (HCC), and evaluate its clinical significance. Methods: Using the term "diaphragm" or "diaphragmatic" and "invasion" or "involvement," we searched for patients in the database of radiologic reports of liver computed tomography or magnetic resonance imaging performed between 2012 and 2016 at our institution. Nine patients with infiltrative invasion of the diaphragm due to recurrent $\mathrm{HCC}$ were included. Their clinical and imaging findings were evaluated.

Results: The median age of patients at the time of diagnosis was 68 years (range, 40 to 73). There were eight men and one woman. Imaging findings of infiltrative invasion of the diaphragm revealed diffuse thickening with enhancement involving a part of the diaphragm. The median interval between initial manifestation on imaging and radiologic diagnosis of infiltrative invasion of the diaphragm was 6.8 months (range, 3.4 to 18.6). In two of three patients who underwent surgical resection, tumors of the diaphragm were controlled without recurrence. In six patients except for one patient who was not followed up, tumors recurred at the resection site or diaphragm tumors showed a partial response or disease progression.

Conclusion: Infiltrative invasion of the diaphragm by recurrent HCC manifested with diffuse thickening and diaphragm enhancement on radiologic imaging. A good prognosis can be expected only in patients who are diagnosed early and undergo surgical resection.
\end{abstract}

Keywords: Carcinoma, hepatocellular; Diaphragm; Recurrence
This is an Open Access article distributed under the terms of the Creative Commons Attribution Non-Commercial License (https:// creativecommons.org/licenses/ by-nc/4.0/). 


\section{INTRODUCTION}

Hepatocellular carcinoma (HCC) is the third leading cause of cancer-related mortality worldwide [1]. Treatments for localized-stage HCC include resection, ablation, or transplantation. Chemoembolization, radiation therapy, and chemotherapy are considered as options for selected patients with unresectable HCC [2]. Although the treatment options for $\mathrm{HCC}$ are evolving, the recurrence rates remain high. Recurrence of HCC can manifest as local tumor progression, occurring in a previously treated site, intrahepatic metastases, or extrahepatic metastases in other organs [3].

Once extrahepatic tumor spread occurs, effective treatment options become limited, and patients are not suitable candidates for curative treatment [4]. Therefore, extrahepatic tumor spread should be detected as it is critical in planning potential therapy and avoiding unnecessary procedures. Moreover, postoperative surveillance is important for managing recurrent tumors and improving patient survival. The main of this surveillance is to identify recurrent HCCs in their earliest stages, thereby enabling the use of curative therapies with better outcomes and decreased mortality [5].

The lungs, abdominal lymph nodes, and bone are the three most common sites of extrahepatic metastases in HCC $[6,7]$. Additionally, metastasis can occur at the adrenal glands, brain, and peritoneum. Other sites such as the diaphragm, and pancreas are considered less common sites of extrahepatic metastases and remain poorly understood [3]. As for the diaphragm, direct invasion by primary HCC is not rare owing to its close location. However, to the best of our knowledge, recurrence of HCC in the diaphragm after surgical resection has only been reported in a single case report to date [8]. In our practice, we have encountered a unique recurrence pattern of HCC: infiltrative invasion of the diaphragm. Therefore, in this study, we aimed to report on infiltrative invasion of the diaphragm, an uncommon manifestation of recurrent $\mathrm{HCC}$, and evaluate its clinical significance.

\section{METHODS}

\section{Patients selection}

The Institutional Review Board (IRB) of Samsung Medical Center approved this retrospective study, and the requirement for patient consent to use clinical data was waived (IRB, file number 2018-01-111). A stepwise search was conducted to identify patients who demonstrated recurrence of HCC, manifesting as diffuse infiltrative invasion of the diaphragm.
First, we searched our radiologic database using the term "diaphragm" or "diaphragmatic" and "invasion" or "involvement" for reports on liver computed tomography (CT) or magnetic resonance imaging (MRI) performed between 2012 and 2016 at our institution. We identified 45 patients who met these criteria. All available radiologic studies were reviewed retrospectively by two non-blinded observers (JHW and KDS) for evidence of recurrent HCC. In all patients in whom recurrent HCC was evidence radiologically, imaging examinations were reexamined to confirm the lack of any distant metastasis at the time of initial treatment and verify that the lesions were new. Thereafter, 36 patients were excluded. Finally, nine patients were included in this study with radiologic diagnosis as gold standard.

\section{Clinical and pathologic data}

The clinicopathological data of all nine patients were retrospectively reviewed to collect the following variables: age, sex, presence of liver cirrhosis, cause of chronic liver disease, Child-Pugh class, levels of tumor markers including serum alpha-fetoprotein and protein induced by vitamin $\mathrm{K}$ absence-II, and initial treatment strategy. The treatment modalities for infiltrative diaphragm invasion and clinical outcomes were also recorded. Pathologic specimens were examined, and findings were reported using Edmondson-Steiner grades [9].

\section{Imaging technique}

CT was performed at our institution using 64-slice or 128slice multidetector CT scanners, including Somatom Definition or Somatom Definition Flash (Siemens Healthineers, Erlangen, Gemany), Toshiba Aquilion detector (Toshiba Medical Systems, Otawara, Japan), and LightSpeed VCT or Discovery CT (GE Healthcare, Chicago, IL, USA). Scanning parameters were as follows: $120 \mathrm{kVp}, 189$ to $200 \mathrm{mAs}$, slice thickness of 2.5 to $3 \mathrm{~mm}$, table speed of 26.5 to $39.4 \mathrm{~mm}$ per rotation (pitch, 0.83 to 1.07), and a single-breath-hold helical acquisition time of 4 to 6 seconds. For contrast-enhanced CT examinations, a total of $120 \mathrm{~mL}$ nonionic iodinated contrast material was administered through the antecubital vein with a power injector at a rate of 3 to $4 \mathrm{~mL} / \mathrm{sec}$. Liver CT examinations were conducted using a multiphasic $\mathrm{CT}$ protocol (contrast-enhanced arterial, portal venous, and delayed phases with or without a non-enhanced image). For the arterial phase (AP), scanning was initiated 30 to 40 seconds after contrast agent injection using a bolus-tracking technique with an automatic power injector. Scanning of the portal and equilibrium phases began at 70 and 180 seconds, respectively, after 
PRECISION AND FUTURE MIEDICINE

Diaphragm invasion in hepatocellular carcinoma

the start of the contrast injection. The scan was performed from the diaphragm to the pubic symphysis.

MRI was performed using a 3.0-T whole-body MRI system (Intera Achieva, Philips Healthcare, Best, the Netherlands) equipped with a dual-source parallel radiofrequency transmission system and a quadrature body coil. Baseline MRI included T1-weighted turbo field-echo in-phase and opposed sequence, breath-hold multishot T2-weighted sequence, and respiratory-triggered single-shot heavily $\mathrm{T2}$-weighted sequence. For gadoxetic acid (Primovist or Eovist, Bayer Schering Pharma, Berlin, Gemany)-enhanced imaging, unenhanced, AP (20 to 35 seconds), portal phase (60 seconds), 3-minute late-phase, and 20-minute hepatobiliary images were obtained using a T1-weighted three-dimensional turbo fieldecho sequence (enhanced T1 high-resolution isotropic volume examination; eTHRIVE, Philips Healthcare). The contrast agent was automatically administered intravenously at a rate of $1 \mathrm{~mL} / \mathrm{sec}$ for a dose of $0.025 \mathrm{mmol} / \mathrm{kg}$ body weight using a power injector, followed by a 20-mL saline flush. Diffusion-weighted single-shot echo-planar imaging with b-values of 0,100 , and $800 \mathrm{sec} / \mathrm{mm}^{2}$ with simultaneous respiratory triggering was performed.

\section{Image analysis}

$\mathrm{CT}$ and MRI findings were reviewed by two radiologists with 4 and 8 years of experience in abdominal imaging interpretation. At the time of initial diagnosis of HCC, the presence of tumor multiplicity, tumor size, location of the tumor, presence of HCC abutting the diaphragm, and presence of distant metastasis were evaluated. The tumor size was determined as the largest diameter on a transverse image, where the equator of the tumor was scanned. Two radiologists then reviewed serial follow-up CT or MRI images to identify patients with diffuse infiltrative invasion of the diaphragm by HCC. The date of diagnosis, date of initial manifestation, location, and size/extent of infiltrative diaphragm invasion as well as response to treatment for this manifestation were recorded. Treatment response was evaluated using the modified Response Evaluation Criteria in Solid Tumors guidelines [10,11].

\section{RESULTS}

The clinical characteristics of patients and tumors on initial imaging are summarized in Table 1 . The median age at diagnosis was 68 years (range, 40 to 73). There were eight men and one woman. Liver cirrhosis was present in six patients (66.7\%). The most common cause of chronic liver disease

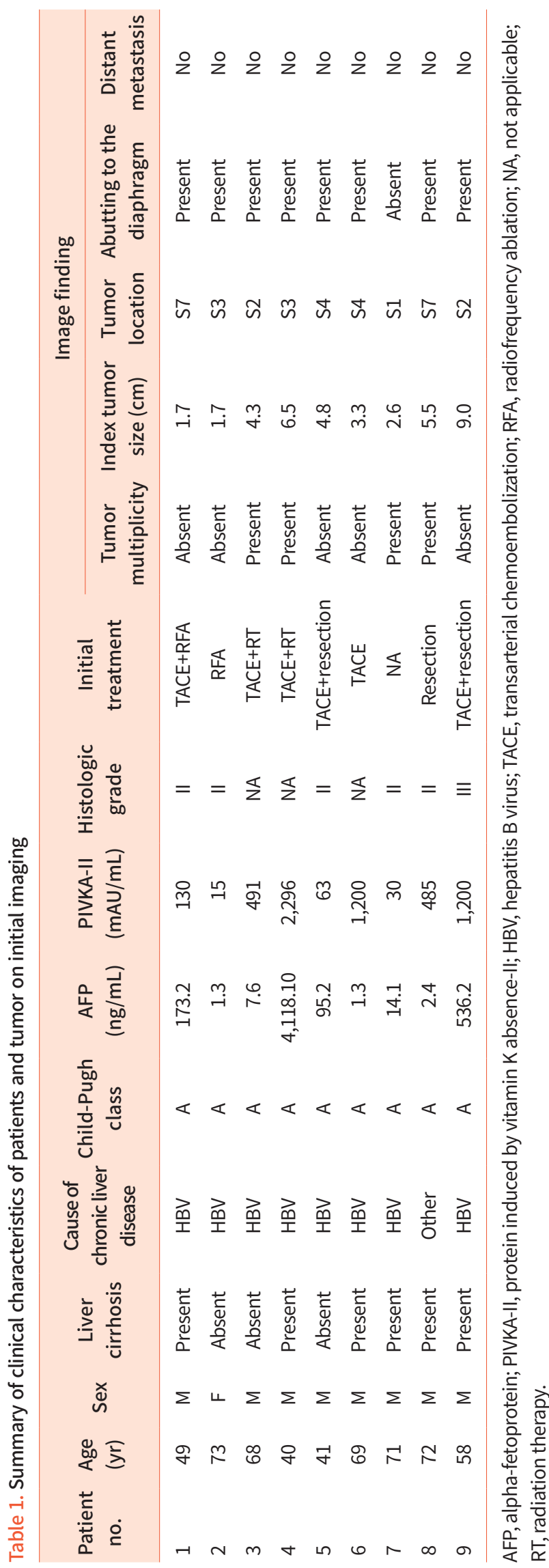


Table 2. Characteristics of recurrent hepatocellular carcinoma manifesting as infiltrative invasion of the diaphragm

\begin{tabular}{|c|c|c|c|c|c|}
\hline $\begin{array}{c}\text { Case } \\
\text { no. }\end{array}$ & $\begin{array}{c}\text { Interval }^{\mathrm{a})} \\
(\mathrm{mo})\end{array}$ & Location & $\begin{array}{c}\text { Size/ } \\
\text { extent } \\
(\mathrm{cm})\end{array}$ & Treatment & Outcome \\
\hline 1 & NA & Rt & 5.3 & Resection & Recurrence \\
\hline 2 & 18.5 & $\mathrm{Lt}$ & 2.8 & Resection & No recurrence \\
\hline 3 & 6.0 & $\mathrm{Lt}$ & 2.8 & Resection+RT & No recurrence \\
\hline 4 & 6.0 & $\mathrm{Lt}$ & 9.6 & Immunotherapy & $\begin{array}{c}\text { Partial } \\
\text { response }\end{array}$ \\
\hline 5 & 6.6 & $\mathrm{Lt}$ & 11.9 & RT & $\begin{array}{c}\text { Partial } \\
\text { response }\end{array}$ \\
\hline 6 & 7.5 & Rt & 2.5 & Sorafenib & Progression \\
\hline 7 & 3.4 & $\mathrm{Lt}$ & 4.9 & Sorafenib & Progression \\
\hline 8 & 18.6 & Rt & 7.7 & Sorafenib & Progression \\
\hline 9 & 7.0 & $\mathrm{Lt}$ & 11.0 & Sorafenib+RT & Progression \\
\hline
\end{tabular}

NA, not applicable; Rt, right; Lt, left; RT, radiation therapy.

${ }^{a}$ Interval between initial manifestation and diagnosis on imaging studies.

was hepatitis B virus infection (88.9\%, 8/9). All patients had well-preserved hepatic function with Child-Pugh class A. At the time of initial diagnosis, the mean index tumor size was $4.3 \mathrm{~cm}$ (range, 1.7 to 9.0). Four patients (44.4\%) had multiple tumors at the time of diagnosis. Eight patients (88.9\%) had HCCs abutting the diaphragm. The patient without this manifestation initially presented with a ruptured HCC that was located in the subcapsular area. None of the patients had distant metastases at the initial staging work-up. Histopathological HCC grades were determined according to the predominant component, and most patients had Edmondson-Steiner grade II disease $(83.3 \%, 5 / 6)$.

Table 2 shows a summary of characteristics of recurrent HCC manifesting as infiltrative invasion of the diaphragm. In eight of the nine patients, the initial manifestation of diffuse infiltrative invasion was localized thickening with an enhancement of the diaphragm (Figs. 1, 2). Later, infiltrative invasion of the diaphragm occurred at the location where the HCCs were previously located. The remaining one patient was treated at another hospital, and follow-up images were not available, making the initial imaging findings of diffuse infiltrative invasion unknown. At the time of diagnosis, the whole or part of one side of the diaphragm was replaced by a tumor, which showed diffuse thickening with an enhancement of the diaphragm. The median interval between initial manifestation on imaging study and diagnosis of infiltrative invasion of the diaphragm was 6.8 months (range, 3.4 to
18.6). The mean size/extent of the tumor involving the diaphragm was $5.3 \mathrm{~cm}$ (range, 2.5 to 11.9 ).

Three patients underwent surgical resection for infiltrative invasion of the diaphragm, and the tumor recurred at the resection site in one patient. Two patients who underwent immunotherapy and radiation therapy showed a partial response. The remaining four patients who underwent sorafenib treatment with or without radiation therapy showed disease progression.

\section{DISCUSSION}

In this study, we report on infiltrative invasion of the diaphragm in cases of recurrent HCCs. Although its incidence is low, it is clinically important to be familiar with this recurrent pattern as the chance of curing it is quite low if the diagnosis is delayed. To the best of our knowledge, this is the first original study to report recurrent HCC manifesting as infiltrative invasion of the diaphragm and its clinical significance.

The median interval between initial manifestation on imaging study and diagnosis of infiltrative invasion of the diaphragm was 6.8 months. In our institution, patients who are treated for $\mathrm{HCC}$ undergo regular follow-up with CT or MRI every 3 months. This suggests that radiologists overlooked the findings of infiltrative invasion of the diaphragm on serial imaging, twice on average; therefore, the diagnosis was delayed. Although we cannot clarify the exact reason for this error, the possible reasons are as follows. First, the diaphragm is considered a less common site of extrahepatic metastasis, and its clinical significance remains poorly understood [6]. Therefore, the radiologists were possibly not familiar with this recurrence pattern. Second, anatomic characteristics of the diaphragm may lead to detection error; it may not be easy to recognize abnormal thickening of the diaphragm on axial images as the diaphragm is parallel to the axial plane. For this reason, thin section and multiplanar reconstruction images have strength in detecting abnormalities of the diaphragm [12]. Extended sequences in MRI, such as diffusion-weighted imaging, may also be useful for detection [13]. Differential point between peritoneal seeding and infiltrative invasion of diaphragm is that, in the case of peritoneal seeding, the diaphragm is relatively intact until it proceeds and invades the diaphragm. However, in the case of direct invasion of diaphragm, diaphragm itself shows enhancing thickening even in minor cases. Coronal image may clearly show the difference.

In eight patients, HCCs were abutting the diaphragm 

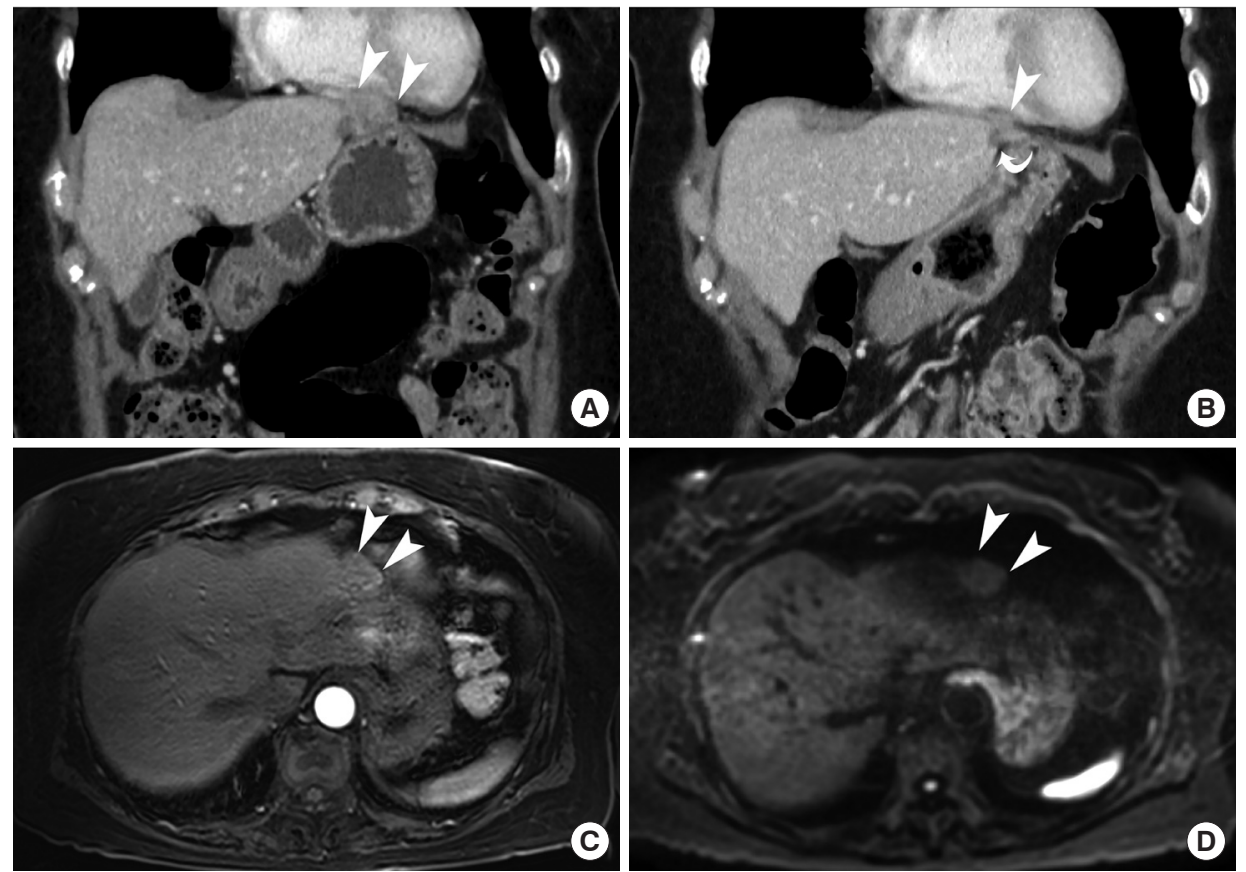

Fig. 1. A 73-year-old man with a history of radiofrequency ablation for hepatocellular carcinoma. Coronal computed tomography (A), (B) shows localized thickening with the enhancement of the diaphragm (arrowheads), which is observed around the previous radiofrequency ablation zone (curved arrow) on coronal computed tomography (B). The extent of thickening of the diaphragm is increased on the 18.5-month follow-up images showing arterial enhancement on gadoxetic acid-enhanced arterial-phase magnetic resonance imaging (arrowheads) (C) and a high signal on diffusion-weighted imaging (arrowheads) (D).

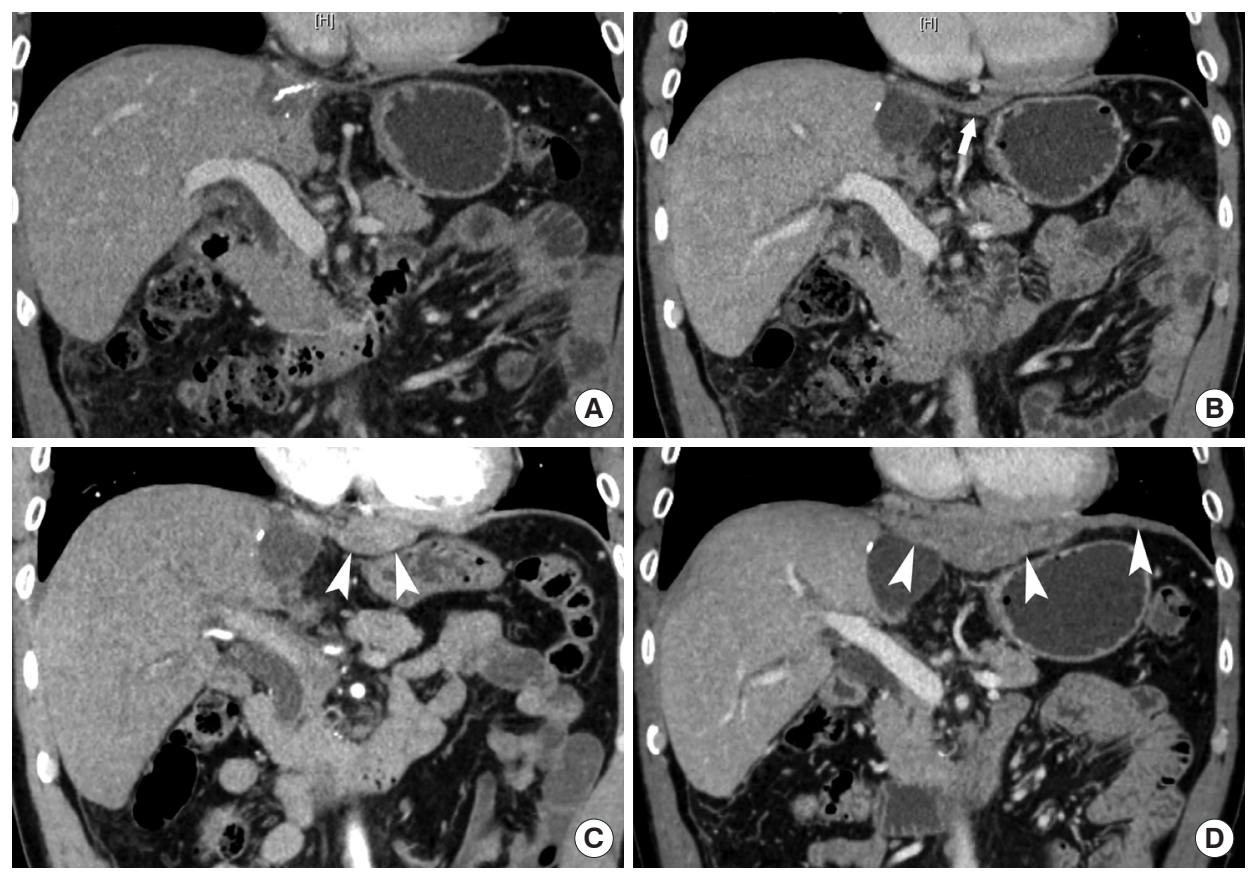

Fig. 2. A 58-year-old man with a ruptured subcapsular hepatocellular carcinoma who underwent transarterial chemoembolization and left lateral sectionectomy. (A) There is no demonstrable abnormal thickening in the diaphragm. (B) Localized thickening of the diaphragm (arrow) can be seen on follow-up computed tomography after 7 months. (C) An increase in the extent of thickening with the enhancement of the diaphragm is observed 4 months later (arrowheads). (D) The extent of thickening of the diaphragm again demonstrates an increase 3 months later, and the central and left diaphragm show diffuse thickening with enhancement (arrowheads). 
where infiltrative invasion occurred later. The remaining patient presented with a ruptured subcapsular HCC, and peritoneal seeding nodules developed at multiple sites, including the subphrenic area. In a previous case report, a similar patient with recurrent HCC in the diaphragm after right hepatic lobectomy for HCC abutting the diaphragm was described [8]. The findings of this and the previous study indicate that infiltrative invasion of the diaphragm is caused by direct invasion of the diaphragm by tumors. For tumors that do not exist in the bare area of the liver, adhesion between the abdominal wall or diaphragm may occur as tumor increase in size. In this case, there is likely a chance for direct invasion of diaphragm. Therefore, the diaphragm should be evaluated cautiously in patients treated for HCCs abutting the diaphragm.

Surgical resection was performed in three patients. In two of them, there was no recurrence at the resection site. However, recurrent tumors developed at the resection site in one patient who had a relatively wide range of tumor invasion compared with the other two patients. None of the patients who underwent non-surgical treatment achieved complete remission. Considering these results, we concluded that early detection in the resectable stage of the disease and prompt surgical resection are important for a good prognosis of infiltrative invasion of the diaphragm by recurrent HCC.

This study had several limitations. First, our study had a retrospective design; therefore, selection bias could exist. Patients might have not been included in this study unless diaphragm invasion or invasion was documented in the radiologic reports. Therefore, we were unable to determine the exact rate of infiltrative invasion of the diaphragm by recurrent HCC. Second, there were no comparable control groups in our study to validate the results. Large scale studies with long-term follow-up are needed to better understand tumor behavior and prognosis. Third, for the ideal study design, pathological diagnosis of diaphragmatic invasion should be used as the standard of reference in all patients. However, in this study radiologic diagnosis was made in five of eight patients $(62.5 \%)$ instead of pathologic diagnosis.

In conclusion, infiltrative invasion of the diaphragm by recurrent HCC manifested as diffuse thickening with an enhancement of the diaphragm. A good prognosis can be expected only in patients who receive an early diagnosis and timely surgical resection.

\section{CONFLICTS OF INTEREST}

No potential conflict of interest relevant to this article was reported.

\section{ORCID}

Chu Hyun Kim https://orcid.org/0000-0002-3515-5163

Kyoung Doo Song https://orcid.org/0000-0002-2767-3622

Jung Han Woo https://orcid.org/0000-0003-4749-9622

\section{AUTHOR CONTRIBUTIONS}

Conception or design: KDS.

Acquisition, analysis, or interpretation of data: CHK, KDS, JHW.

Drafting the work or revising: CHK, KDS.

Final approval of the manuscript: $\mathrm{CHK}$, KDS.

\section{REFERENCES}

1. Parkin DM, Bray F, Ferlay J, Pisani P. Estimating the world cancer burden: Globocan 2000. Int J Cancer 2001;94:1536.

2. Altekruse SF, McGlynn KA, Reichman ME. Hepatocellular carcinoma incidence, mortality, and survival trends in the United States from 1975 to 2005. J Clin Oncol 2009; 27:1485-91.

3. Shah SA, Cleary SP, Wei AC, Yang I, Taylor BR, Hemming $A W$, et al. Recurrence after liver resection for hepatocellular carcinoma: risk factors, treatment, and outcomes. Surgery 2007;141:330-9.

4. Yang $\mathrm{Y}$, Nagano $\mathrm{H}$, Ota $\mathrm{H}$, Morimoto $\mathrm{O}$, Nakamura $\mathrm{M}$, Wada $\mathrm{H}$, et al. Patterns and clinicopathologic features of extrahepatic recurrence of hepatocellular carcinoma after curative resection. Surgery 2007;141:196-202.

5. van Meer S, de Man RA, Coenraad MJ, Sprengers D, van Nieuwkerk KM, Klumpen HJ, et al. Surveillance for hepatocellular carcinoma is associated with increased survival: results from a large cohort in the Netherlands. J Hepatol 2015;63:1156-63.

6. Katyal S, Oliver JH 3rd, Peterson MS, Ferris JV, Carr BS, Baron RL. Extrahepatic metastases of hepatocellular carcinoma. Radiology 2000;216:698-703.

7. Natsuizaka M, Omura T, Akaike T, Kuwata Y, Yamazaki K, Sato T, et al. Clinical features of hepatocellular carcinoma with extrahepatic metastases. J Gastroenterol Hepatol 
2005;20:1781-7.

8. Ueda J, Yoshida H, Mamada Y, Taniai N, Mineta S, Yoshioka $\mathrm{M}$, et al. Resection of hepatocellular carcinoma recurring in the diaphragm after right hepatic lobectomy. J Nippon Med Sch 2011;78:30-3.

9. Edmondson HA, Steiner PE. Primary carcinoma of the liver: a study of 100 cases among 48,900 necropsies. Cancer 1954;7:462-503.

10. Llovet JM, Di Bisceglie AM, Bruix J, Kramer BS, Lencioni R, Zhu AX, et al. Design and endpoints of clinical trials in he- patocellular carcinoma. J Natl Cancer Inst 2008;100:698711.

11. Lencioni R, Llovet JM. Modified RECIST (mRECIST) assessment for hepatocellular carcinoma. Semin Liver Dis 2010;30:52-60.

12. Desir A, Ghaye B. CT of blunt diaphragmatic rupture. Radiographics 2012;32:477-98.

13. Nason LK, Walker CM, McNeeley MF, Burivong W, Fligner CL, Godwin JD. Imaging of the diaphragm: anatomy and function. Radiographics 2012;32:E51-70. 